\title{
Phlegmasia Cerulea Dolens after Ovarian Cancer Treatment with Bevacizumab: A Case Report
}

Belmaachi Abderrafie*, Sara Ridai, Hassan Jouhadi, Souha Sahraoui, Zineb Bouchbika, Nadia Benchakroun, Nezha Tawfiq, Abdellatif Benider

Centre Mohammed VI de traitement des Cancers, CHU Ibn Rochd Faculté de Médecine et Pharmacie, Université Hassan II, Casablanca, Morocco

DOI: $10.36347 /$ sjmcr.2020.v08i08.004

| Received: 03.07.2020 | Accepted: 15.07.2020 | Published: 08.08.2020

*Corresponding author: Belmaachi Abderrafie

\section{Abstract}

Bevacizumab has been shown to improve progression-free survival in women with ovarian cancer in multiple clinical trials. However, serious adverse effects, such deep vein thromobosis, can occur [1]. Phlegmasia cerulea dolens is a rare entity secondary to grave Deep Vein Thrombosis (DVT), in the lower extremities, and associated with rapidly progressive pain and marked swelling that may compromise the perfusion of the limb. This situation may lead to venous gangrene and the limb's amputation secondary to a compartment syndrome [2]. We present the case of a 45year-old female who presented eight days after the fourth administration of bevacizumab for ovarian cancer treatment, a bilateral leg pain with progressive edema which also began to exhibitwith a black discoloration. Venous dopplers showed bilateral occlusions of the common femoral, saphenofemoral, superficial femoral, popliteal, posterior tibial and peroneal veins. To our knowledge, this is the first case with bevacizumab associated Phlegmasia cerulea dolens .

Keywords: Bevacizumab, Phlegmasia cerulea dolens (PCD), deep Thrombosis, ovarian cancer.

Copyright @ 2020: This is an open-access article distributed under the terms of the Creative Commons Attribution license which permits unrestricted use, distribution, and reproduction in any medium for non-commercial use (NonCommercial, or CC-BY-NC) provided the original author and source are credited.

\section{INTRODUCTION}

Bevacizumab is a vascular endothelial growth factor (VEGF) inhibitor that has improved survival rates among patients with cancers of lung, colon and glioblastomas. Bevacizumab has been associated with arterial and venous thromboembolism occurring during the treatment period [3].

Cancer patients often have a hypercoagulable state due to the production of substances with procoagulant activity like cancer procoagulant, proinflammatory and proangiogenic cytokines and upregulation of tissue factor expression on tumor cells, endothelial cells, and circulating monocytes [4]. The risk of venous thrombosis in cancer patients is also increased due to various other factors including chemotherapeutic drugs, surgery, hospitalization and placement of central venous catheters.

Phelgmasia cerulea dolens (PCD) is a rare cause of shock that can complicate deep venous thrombosis and carries a high risk of mortality it can occur at any age, although its peak incidence is set between the 5th and 6th decade of life. PCD is where there is a total occlusion of the affected vein, impaired collateral venous system with arterial occlusion caused by the subsequent compartment syndrome. Hence, these patients present with a classical clinical trial of; intense pain out of proportion, significant edema $\&$ cyanosis of the affected limb. From which comes the name phlegmasia cerulean dolens (blue, painful leg). It is associated with a high risk of amputation (50\%), pulmonary embolism $(22 \%)$ and a mortality rate up to $40 \%[2,4]$. Because of the different involvement of the venous and arterial systems of the affected limb in Phlegmasia Cerulea Dolens \& Phlegmasia Alba Dolens the presentation, management and prognosis of each one of them is also different from each other. Several treatment options have been suggested to reduce the high rate of morbidity and mortality, but due to the rarity of this condition a gold standard treatment plan is still controversial.

\section{Case Presentation}

A 48-year-old female without known allergies, or significant pathological history, treated for a high grade mixed type ovarian adenocarcinoma receiving palliative therapy with gemcitabine-bevacizumab for her platinum-resistant recurrent epithelial ovarian cancer. At a follow-up visit after three cycles of the new therapy. 
She notices progressive edema of of the two lower inferior members, with pain. Given these symptoms, she decides to turn to the emergency room at the "CHU IBN ROCHD" Hospital in CASABLANCA, Maroc. She had a marked swelling of the lower limb which obliged the medical personnel to remove her clothes with scissors before attending her; she was afebrile and hemodynamically stables, with $125 \mathrm{bpm}$ and a systolic pressure of $70 \mathrm{mmHg}$ on presentation, without cardiac arrhythmia, no dyspnea or chest pain. Arterial pulses were present and symmetric in both lower extremities, there was lower limb edema without difference in diameter compared to the contralateral limb with severe cyanosis, there was no infection signs (Fig1). With a diagnosis of severe Deep Vein Thrombosis (DVT) associated to phlegmasia, anticoagulation with LMWH and complete rest in the Trendelenburg position were indicated.

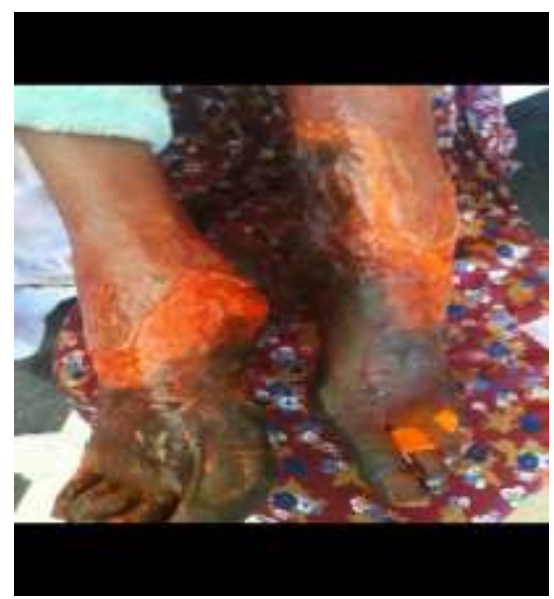

Fig-1: Lower extremity oedema with discolouration and cyanosis.

CT angiogram of chest and CT venogram lower limbs showed massive bilateral pulmonary emboli in the territory of the two pulmonary artery. The echocardiography detected a right ventricular dilatation with normal left function. CT abdomen revealed a large tumor masses, peritoneal carcinosis and ascities (Fig 2). Lower extremity ultrasound confirmed acute bilateral DVTs involving of the common femoral, saphenofemoral, superficial femoral, popliteal, posterior tibial and peroneal veins. Laboratory evaluations excluded thrombophilia, heparin- induced thrombocytopenia and thrombotic thrombocytopenic purpura, the patient was initiated on anticoagulation with unfractionated heparin and supportive care such as lower limb elevation and analgesics. Her eventual work-up for malignancy and blood cultures were negative. Despite 24 hours of anticoagulation, her pain and swelling did not subside. The patient was diagnosed as a case of 'Phlegmasia Cerulea Dolens' of left leg, compartment syndrome with pulmonary embolism (PE).

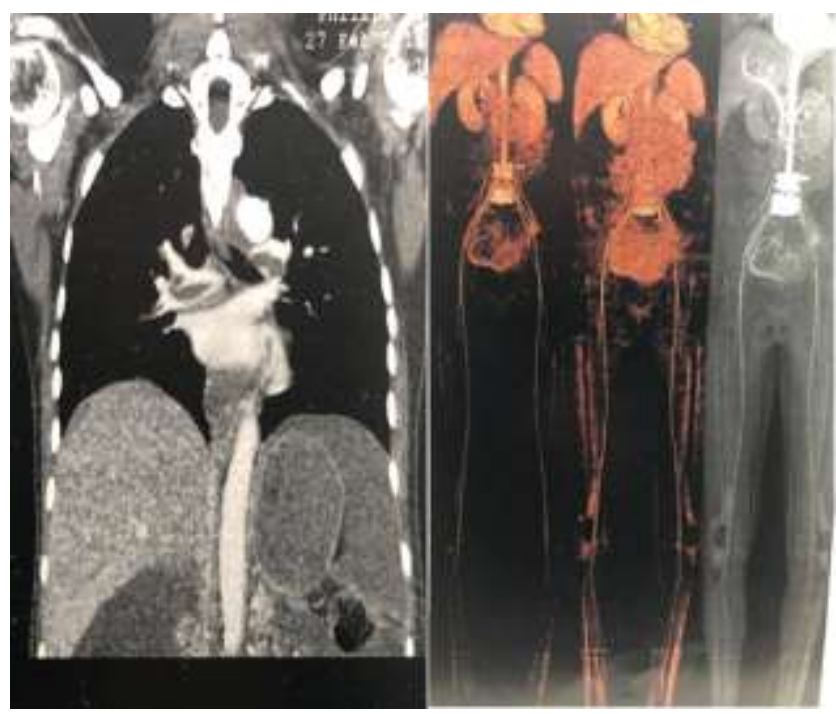

Fig-2: Thrombosis of pulmonary artery extending to its bifurcation and the two branches

The patient evolves favorably, having resolved the edema, the pain and the perfusion of the limb, after three weeks of oral anticoagulation and elastic compression stockings(Fig:3). 


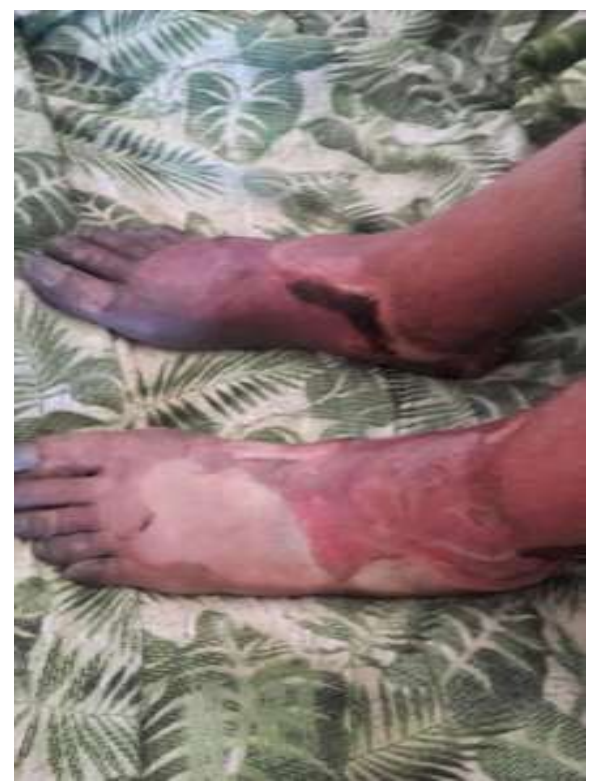

Fig-3: Favorable evolution with resolved oedema, pain and limb perfusion

After one month of anticoagulation the patient presented dyspnea and chest pain, she died one day after her admission in intensive care in emergency unit, following pulmonary emboli complications.

\section{DISCUSION}

Bevacizumab (Avastin) is a humanized monoclonal antibody that is approved by the U.S. Food and Drug Administration for the treatment of patients with colon cancer, lung cancer, glioblastoma, renal-cell carcinoma, and age-related macular degeneration. As angiogenesis inhibitor, Bevacizumab blocks angiogenesis by inhibiting vascular endothelial growth factor A (VEGF-A).

Bevacizumab prevents VEGF ligand-receptor binding and down-streams signaling [3]. Anti-VEGF inhibits tumor angiogenesis and causes the normalization of surviving tumor vasculature and the regression of existing immature tumor vasculature [3, 5]. Bevacizumab can cause arterial thrombosis, recurrent PRES (posterior reversible encephalopathy syndrome) and venous thromboembolism [3]. Shobha Rani Nalluri et al. performed a meta-analysis of 7,956 patients from 15 phase II and II randomized clinical trial (bevacizumab, $\mathrm{n}=4,292$; control, $\mathrm{n}=3,664$ ) and concluded that bevacizumab increased the risk of VTE, both with high and low doses and the risk vary with tumor type and used chemotherapy.

Phlegmasia cerulea dolens (PCD) is a rare, it represent a severe form of DVT that results in massive thrombosis of the deep venous system in a lower limb. Its incidence is not known. Cancer, hypercoagulability and previous surgery can be risk factors.

Clinical findings and physical exam are the first step in the process of early diagnosis. Venous dopplers can help to confirm diagnosis, and estimate the severity and the exent. CT angiogram of chest is necessary to show the pulmonary emboli [2].

Due to its rarity, there is no consensus regarding its management, Treatment of phlegmasia should be started as soon as possible. Medical treatment includes anticoagulation with an elevation of the affected limb. Recent literature suggests that subcutaneous low-molecular-weight heparins such as Enoxaparin [6] or Fondaparinux [7] are safe and effective in the treatment of PCD. New oral anticoagulants, Factor Xa inhibitors (rivaroxaban, apixaban, edoxaban) and oral direct thrombin inhibitors (dabigatran) can be prescribed in DVT, but are not suitable for PCD.

Surgical treatment of PCD involves endovascular intervention or open surgical thrombectomy. Endovascular targeted thrombolytic therapy is the intervention of choice and has been proven to be effective, and safe [6]. Absolute contraindications for endovascular thrombolysis include recent head trauma, recent cerebrovascular accident (less than two months), and severe hypertension, allergy to thrombolytic agents or active bleeding in a noncompressible space.

\section{Conclusion}

Bevacizumab is considered a safe therapy in case of ovarian cancer, but some serious complications, such as thrombosis can occur.Phlegmasia Cerulea Dolens is a severe form of deep vein thrombosis with a high rate of mortality and morbidity. An early detection and appropriate decision regarding the line of management is crucial to save the patient and to preserve the limb and its function. 


\section{Conflict of interests} of interest.

The authors declare that they have no conflicts

\section{REFERENCES}

1. Martin JY, Urban RR, Liao JB, Goff BA. Bevacizumab toxicity in heavily pretreated recurrent epithelial ovarian, fallopian tube, and primary peritoneal cancers. Journal of gynecologic oncology. 2016 May 3;27(5).

2. Chinsakchai K, ten Duis K, Moll FL, de Borst GJ. Trends in management of phlegmasia cerulea dolens. Vascular and endovascular surgery. 2011 Jan;45(1):5-14.

3. Suenaga M, Mizunuma N, Kobayashi K, Shinozaki E, Matsusaka S, Chin K, Kuboki Y, Ichimura T, Ozaka M, Ogura M, Fujiwara Y. Management of venous thromboembolism in colorectal cancer patients treated with bevacizumab. Medical Oncology. 2010 Sep 1;27(3):807-14.
4. Hasegawa S, Aoyama T, Kakinoki R, Toguchida J, Nakamura T. Bilateral phlegmasia dolens associated with Trousseau's syndrome: a case report. Archives of physical medicine and rehabilitation. 2008 Jun 1;89(6):1187-90.

5. Nalluri SR, Chu D, Keresztes R, Zhu X, Wu S. Risk of venous thromboembolism with the angiogenesis inhibitor bevacizumab in cancer patients: a meta-analysis. Jama. 2008 Nov 19;300(19):2277-85.

6. Oguzkurt L, Ozkan U, Demirturk OS, Gur S. Endovascular treatment of phlegmasia cerulea dolens with impending venous gangrene: manual aspiration thrombectomy as the first-line thrombus removal method. Cardiovascular and interventional radiology. 2011 Dec 1;34(6):1214-21.

7. Erdoes LS, Ezell JB, Myers SI, Hogan MB, LeSar CJ, Sprouse LR. Pharmacomechanical thrombolysis for phlegmasia cerulea dolens. The American Surgeon. 2011 Dec 1;77(12):1606-12. 